\title{
Comparison of the Recurrence Rate of Entropion via Subciliary Route and Transconjunctival Route in Combined Lateral Tarsal Strip with Retractor Advancement Procedure for Involutional Entropion Correction
}

\author{
Faisal Aziz Khan, Mohammed Farooq Hyder, Shafaq Pervez Khan Niazi and Umair Tariq Mirza \\ Department of Eye, Combined Military Hospital, Jhelum, Pakistan
}

\begin{abstract}
Objective: To compare the recurrence rate of entropion via subciliary route and transconjunctival route in combined lateral tarsal strip with retractor advancement procedure for involutional entropion correction.

Study Design: Quasi-experimental study.

Place and Duration of Study: Department of Eye, Combined Military Hospital, Jhelum Cantt, Pakistan from March 2018 to November 2020.

Methodology: Seventy-eight eyes of 78 patients with entropion were randomly divided into two groups of 39 each. One group underwent a lateral tarsal strip procedure combined with retractor advancement through the subciliary approach; and the second group underwent the same procedure through the transconjunctival approach. A strip of preseptal orbicularis was excised in every case. Recurrence rate of entropion was recorded in both groups at six-month postoperation. Duration of surgery was recorded in each case. Fisher's Exact test was selected to compare the recurrence rate of entropion and independent samples ' $\mathrm{t}$ ' test was elected for comparing duration of surgery among the two groups.

Results: The recurrence rate of entropion with transconjunctival route repair was statistically not significant compared with the subciliary route repair $(p>0.999)$. The transconjunctival route repair achieved statistically significant shorter duration of surgical time as compared to the subciliary route $(p<0.001)$.

Conclusion: The transconjunctival approach for involutional entropion correction is as effective as the subciliary approach with lateral tarsal strip and retractor advancement procedure. Surgery duration through the transconjunctival route is significantly shorter as compared to the subciliary approach.
\end{abstract}

Key Words: Entropion, Involutional, Lateral tarsal strip, Lower eyelid, Retractor advancement, Recurrence, Subciliary, Transconjunctival.

How to cite this article: Khan FA, Hyder MF, Niazi SPK, Mirza UT. Comparison of the Recurrence Rate of Entropion via Subciliary Route and Transconjunctival Route in Combined Lateral Tarsal Strip with Retractor Advancement Procedure for Involutional Entropion Correction. J Coll Physicians Surg Pak 2021; 31(04):429-433.

\section{INTRODUCTION}

Involutional entropion is the most common type of entropion. ${ }^{1}$ Classically, the causes of involutional entropion are horizontal laxity of the lower eyelid, overriding of the orbicularis oculi muscle and disinsertion or attenuation of the lower eyelid retractors. $^{2}$

Correspondence to: Dr. Faisal Aziz Khan, Department of Eye, Combined Military Hospital, Jhelum, Pakistan

E-mail: faisaleyecare@gmail.com

Received: December 20, 2020; Revised: February 13, 2021;

Accepted: March 05, 2021

DOI: https://doi.org/10.29271/jcpsp.2021.04.429
To correct involutional entropion, surgical procedure is aimed at the repair of these primary anatomic defects. ${ }^{3}$ The choice of surgical procedure is based upon each patient's condition such as the presence of vertical laxity, horizontal lid laxity, and overriding of the orbicularis muscle. ${ }^{4}$

Various surgical procedures have been advocated to correct lower eyelid involutional entropion. ${ }^{4}$ It is very common practice to see the presence of significant both horizontal as well as vertical lid laxity in the same patient. ${ }^{3}$ In such common scenarios, in addition to retractor repair, it becomes necessary to achieve horizontal lid shortening by doing either lateral canthal ligament tucking, full-thickness pentagonal wedge resection, or lateral tarsal strip (LTS) fixation. ${ }^{3}$

The mostimportantcomplication of surgical correction of involu- 
tional entropion is recurrence. ${ }^{5}$ The recurrence rate is further decreased when combination procedures are performed to correct the multiple causative factors involved. ${ }^{4}$ Utilising the LTS procedure for correcting the horizontal lid laxity and combining it with Jones retractor plication procedure, gives longer lasting correction of entropion. ${ }^{6}$

Both subciliary and transconjunctival approaches have been described in literature for repairing involutional entropion. Either of these approaches can be utilised to perform combination procedures to correct horizontal laxity, prolapse of orbital fat, overriding of preseptal orbicularis oculi and vertical laxity. ${ }^{1,7}$

The transconjunctival approach to lower lid surgery has gained popularity over the subciliary approach because of its inconspicuous scar. ${ }^{8,9}$ Moreover subciliary approach also restores the normal lid anatomy after surgical correction of involutional entropion. ${ }^{9}$ The risk of developing overcorrection and lower lid retraction with subciliary entropion repair approach can be outmanoeuvered by the transconjunctival route. ${ }^{10}$

Reviews of previous published literature showed that there was no study to date, both locally and internationally, that has compared recurrence of entropion after a combined procedure of lateral tarsal strip with retractor advancement (RA), using transconjunctival and subciliary approaches.

Therefore, the objective of this study was to compare the recurrence rate of entropion via subciliary route and transconjunctival route in combined lateral tarsal strip with retractor advancement procedure for involutional entropion correction.

\section{METHODOLOGY}

This prospective randomised controlled trial was conducted at the Eye Department, Combined Military Hospital, Jhelum, Pakistan from March 2018 to November 2020. The study was approved by the Ethical Review Committee of the Hospital. Patients attending outpatient unit of Eye Department were selected. The inclusion criteria was primary involutional lower eyelid entropion with significant horizontal lid laxity, minimal dermatochalasis, mild fat protrusion and cooperative patients who were fit for local anaesthesia. The exclusion criteria were elderly patients (age $>70$ years) with concomitant medical problems, on-going anticoagulant therapy, bleeding diathesis, and previous eyelid surgery. A sample size of 39/51 ( $n=39 / 51)$ eyes in each group was calculated to detect a significant difference in the recurrence rate between the subciliary and transconjunctival group with $80-90 \%$ power and a two-sided $\alpha$ of 0.05 , using recurrence rate of $0.00 \%{ }^{11}$ (P1=recurrence rate via subciliary route) and $18.2 \%{ }^{9}(\mathrm{P} 2=$ recurrence rate via transconjunctival route) from previously published data utilising similar surgical techniques with the help of WHO calculator. Eyes of 80/102 patients were selected and randomly allocated into two equal groups labeled as subciliary (SC group) and transconjunctival (TC group) of 40/51 each, based on computer generated random numbers table. SC group patients underwent subciliary retractor advancement (RA) with lateral tarsal strip (LTS) and TC group patients underwent same procedure via transconjunctival route.
Written informed consents were obtained from all patients. After obtaining relevant ocular and systemic history, each patient underwent a careful naked eye and slit lamp examination. The eyelids were inspected by asking patient to open and close, forcibly close and move eyes in down gaze. On palpation, the laxity of medial and lateral canthal tendon was assessed. Snap back test, eyelid eversion and eyelid distraction tests were performed. After obtaining permission, all patients were photographed. All surgeries were performed by a single surgeon, under local anaesthesia. IV sedation was employed selectively by first author. Duration of surgical procedure was recorded for every patient, beginning from the start of incision till the last completion of suture. In the subciliary group, skin incision was marked $3-4 \mathrm{~mm}$ below the lash line, extending from below the punctum to the lateral canthus. A 50:50 $2 \mathrm{ml}$ mixture of lignocaine with adrenaline plus bupivacaine was infiltrated subcutaneously along the marked skin crease as well as at lateral canthal skin and inner aspect of orbital rim against the bone. Skin was incised with No. 15 blade along the skin marking. Orbicularis muscle was incised and orbital septum opened. Fat pads and retractor layer was identified. Fat pads were retracted and dissected free from the underlying retractor layer. The retractor layer was identified for dehiscence or attenuation. In case of attenuation, retractors were disinserted 1-2 $\mathrm{mm}$ below lower border of tarsus. In either case, they were reattached using 3 interrupted 5-0 vicryl sutures from the retractor edge to the inferior tarsal edge. Lid margin was assessed by asking patient to look up and down. If lid margin looked markedly overcorrected, or undercorrected then position of retractor suture bites were adjusted, accordingly. A strip of preseptal orbicularis muscle was excised. Next, a lateral tarsal strip procedure was performed as described later. The subciliary incision was closed with continuous sutures and lateral canthotomy with interrupted 6-0 vicryl. In the TC group, local anaesthetic, as described above, was injected at the lateral canthal skin, orbital rim against the bone and subconjunctivally with $30 \mathrm{G}$ needle $1-2 \mathrm{~mm}$ below the tarsal margin. First, a lateral canthotomy and inferior cantholysis was done. Then, using monopolar Colorado needle conjunctiva and lower lid retractors were both cut $1-2 \mathrm{~mm}$ below tarsal border along full horizontal extent. Retractors were separated from the anterior lying fat pads for $4-5 \mathrm{~mm}$ as well as from the posterior conjunctiva to create a free edge. A strip of preseptal orbicularis was excised. Retractors were reattached to the anterior inferior tarsal border with four interrupted 5-0 vicryl sutures. In both groups, after retractors were attached, the lateral tarsal strip was completed by splitting the lid margin along grey line, fashioning a tarsal strip, excising the tarsal plate after drawing it to the orbital rim and reattaching it with 5-0 vicryl suture inside the orbital rim just inferior to superior crus of lateral canthal tendon. The redundant anterior lamella was excised. Subciliary incision was closed with continuous 5-0 vicryl sutures and lateral canthotomy incision was closed with interrupted 5-0 vicryl sutures. A topical antibiotic eye ointment was placed and prescribed three times a day for two weeks. Follow-up was scheduled at 01,03 and 06 month postoperatively. At each follow-up, surgical outcomes and 
complications including recurrence, residual entropion, over correction, infection, lid contour abnormalities and lateral canthal angle displacement were recorded. Success was defined as restoration of normal anatomical position of lid margin and no inward turning of lid margin on eyelid closure.

Statistical analysis was performed using Statistical Package for Social Sciences (SPSS) version 20.0 for Windows. Descriptive statistics, mean and standard deviation (S.D), and normality test (Kolmogorov-Smirnov) were computed for age, duration of surgery in each group. The age of patients and duration of surgery among the two groups were compared using independent sample t-test. Recurrence of entropion was described as frequency and percentage. Fisher's Exact test was selected to compare the recurrence rate of entropion between the two groups. The test was 2 -tailed. A p-value $<0.05$ was considered statistically significant

\section{RESULTS}

The study cohort comprised of 78 eyes of 78 patients. A total of 80 procedures were performed with 40 cases undergoing retractor advancement (RA) with lateral tarsal strip (LTS) in each group. Out of these 80 procedures, two patients (one from each group) did not report for follow- up after the first visit, and were excluded from the study cohort.

The age ranged from 55-80 years. Mean age in SC group was $64.62 \pm 5.58$ years, and in TC group was $64.56 \pm 4.96$ years. Both groups were found to be age-matched as there was no statistical difference in the mean ages of two groups $(p=0.966)$. Out of the 78 patients, $55(70.5 \%)$ were males and $23(29.5 \%)$ were females. All 78 patients completed their 12-month follow-up.

A statistically significant shorter duration of surgical time was detected in patients, who underwent LTS plus RA through the conjunctival route as compared to those patients, who underwent the same procedure with the skin approach as shown in Table I. Both transconjunctival and subciliary approaches depicted statistically insignificant difference in the recurrence rates of entropion as shown in Table I.

Table l: Comparison of the recurrence rate of entropion and surgery duration among subciliary and transconjunctival groups $(n=78)$.

\begin{tabular}{|l|c|c|}
\hline $\begin{array}{l}\text { Subciliary group value } \\
(\mathbf{n}=\mathbf{3 9})\end{array}$ & $\begin{array}{c}\text { Transconjunctival } \\
\text { group }\end{array}$ & $\begin{array}{c}\mathbf{p} \text {-value } \\
(\mathbf{n}=\mathbf{3 9})\end{array}$ \\
\hline Recurrence rate $1(2.6 \%)$ & $2(5.1 \%)$ & $p>0.999$ \\
\hline $\begin{array}{l}\text { Surgical time (mean } \pm \text { SD) } \\
64.92 \pm 4.68 \text { minutes }\end{array}$ & $45.13 \pm 3.33$ minutes & $p<0.001$ \\
\hline
\end{tabular}

Nomajorintraoperativecomplications were recorded. Postoperatively in the SC group, 02 (5.1\%) patients developed ectropion, while no ectropion was seen in TC group at the 12-month followup; and this difference was statistically insignificant $(p=0.494$. One (2.6\%) patient had stitch abscess and 02 (5.1\%) developed lateral canthal dystopia in SC; and in TC group, 03 (7.7\%) patients had mild conjunctivochalasis and 01 (2.6\%) patient showed lateral canthal dystopia. Three (7.7\%) patients among the SC group complained of a visible scar in the infraciliary region.

\section{DISCUSSION}

Among all the complications of involutional entropion repair, recurrence is the major concern. ${ }^{12}$ Intraoperatively, recurrence is determined by the choice of surgical procedure as well as whether a single procedure or combination of surgical procedures is performed. ${ }^{4,13}$ Elias et al. concluded that horizontal component is more important than the vertical component for correcting entropion; and suggested that it is far more important to tighten the lid horizontally by employing LTS procedure even when horizontal laxity is not clinically detectable. ${ }^{14}$ LTS is a very popular procedure for correcting horizontal lid laxity. ${ }^{14-16}$ Consequently, the authors chose LTS procedure for correcting horizontal lid tightening in each case.

Various combinations of surgical procedures have been published for minimising recurrences after entropion repair. Many surgeons have combined retractor advancement (RA) with lateral tarsal strip (LTS). ${ }^{17,18}$ Some have combined RA with wedge resection. ${ }^{5,17}$ Others have combined everting sutures with LTS procedure. ${ }^{14,18,19}$ Review of literature shows that recurrence rates of combining LTS with RA or wedge resection with RA is low as compared to single procedure and ranges from as low as $0 \%$ in multiple studies ${ }^{11}$ to $18.2 \%$; ${ }^{17}$ whereas, with single procedure such as LTS and RA, the recurrence rates in preexisting horizontal lid laxity varies from $8.7 \%$ to as high as $30 \%{ }^{11,17}$ The reason behind high recurrence rates with single procedure is its inability to correct all the three anatomical causative factors simultaneously namely, vertical laxity, horizontal laxity, and orbicularis override. ${ }^{4,14} \mathrm{~A}$ combination procedure was elected to keep the recurrence rates low as mentioned in previously published data. ${ }^{11}$

LTS plus RA procedure is advantageous over the wedge resection plus RA procedure in that it provides better cosmesis, maintains normal anatomy of lid margin and avoids the potential complications of lid notching ${ }^{16,20}$ and wound dehiscence, which may occur after wedge resection. ${ }^{5,16}$ Unlike LTS, wedge resection may not be very effective in significant lateral canthal tendon laxity ${ }^{17}$ as it adds strain on the already weakened lateral canthal tendon. ${ }^{20}$ Thus keeping in view the anatomical abnormalities, the authors repaired every case by combining RA with LTS procedure as they show very late recurrence with high patient satisfaction. 6,14

Overriding of the orbicularis oculi muscle is a common finding in involutional entropion. ${ }^{4,5}$ This overriding may be the consequence of anatomical changes of dehiscence between anterior and posterior lamella, causing the strong preseptal orbicularis to override the pretarsal muscle. ${ }^{21}$ Nevertheless, many studies have emphasised that orbicularis myectomy be performed during entropion surgery as it is an anatomical abnormality. ${ }^{1,17}$ Mahmoud et al. suggested that excising a strip of preseptal orbicularis improves appositional strength of the lid during eyelid closure; ${ }^{22}$ and resultant scarring after excision, creates a barrier between pretarsal and preseptal orbicularis thus preventing override. ${ }^{2,20}$ In this study, to improve the success 
rate of surgery, a strip of preseptal orbicularis muscle was excised in every case regardless of preoperative finding of muscle override.

Traditionally, entropion has been repaired via the skin approach. The transconjunctival route is ideal as it avoids a subciliary incision; since in oculoplastic surgery, cosmesis is as important as main outcome of surgery. Therefore, one should consider the cosmetic after effect before choosing the appropriate approach. ${ }^{20}$ Additionally, the transconjunctival route for entropion surgery addresses all the correctable causes of senile entropion, except when there is considerable dermatochalasis and festoons. ${ }^{23}$ Henceforth, the authors did not select eyelids with dermatochalasis in this cohort, since dermatochalasis is a possible causative factor for entropion. ${ }^{14}$ In this study, 03 (7.7\%) patients reported a noticeable scar that underwent entropion repair via skin approach. Therefore, the authors recommend that transconjunctival approach should be the first choice for entropion repair as it is exceedingly gratifying for both the surgeon and patient.

Previous studies have recorded a higheroccurrence of postoperative entropion via transconjunctival route, but it has been statistically insignificant compared to skin approach. ${ }^{9}$ Conversely, there is a higher incidence of lid retraction and overcorrection with the subciliary approach. ${ }^{18}$ In this study, none of the patients developed lid retraction or overcorrection in the TC group; whereas, 02 (5.1\%) patients developed ectropion in SC group. Review of published data on recurrence of entropion after LTS plus RA procedure shows that in the subciliary approach, the rate ranges from $0.0 \%$ to $8.7 \%{ }^{17}$; and in transconjunctival approach, it varies from $15 \%$ to $18.2 \%$. ${ }^{17,24}$ However, none of these researches have compared both approaches together in one study. This study is unique in this aspect that the authors conducted a randomised controlled trial to compare both approaches. There was a statistically insignificant difference in recurrence incidence among the two approaches, emphasising that the transconjunctival approach, is as effective as the subciliary approach.

The success of a surgical procedure is also judged by the level of patient comfort during surgery. ${ }^{3}$ In the context of patient comfort, after performing this procedure through the conjunctival route, it was found that the transconjunctival route produced significantly shorter duration of surgical time of 45.13 \pm 3.33 mins, compared to $64.92 \pm 4.68$ minutes with subciliary approach. Kreis et al. also concluded that correcting entropion through transconjunctival route is time-efficient and effective as well; ${ }^{9}$ whereas, with transcutaenous approach Goel et al. reported an average surgical time of 66 minutes for LTS plus $\mathrm{RA}^{25}$ The shorter surgical time through conjunctiva was the result of swift conjunctival incision with monopolar Colorado needle, no prerequisite for a orbicularis incision, immediate identification of retractors beneath the conjunctiva, and no compulsion for conjunctival suture wound closure at the end of surgery.

The question of whether to use a nonabsorbable or absorbable suture for reattaching the tarsal strip and retractors to the periosteum and inferior tarsal border respectively also varies among various authors. ${ }^{17,25}$ There is no study to date that has analysed whether nonabsorbable suture is advantageous over the absorbable suture material in providing long term correction of horizontal and vertical laxity. In this study, 5-0 vicryl sutures in every case for both retractor and tarsal strip reattachment. Their effect on long term success necessitates future studies.

\section{CONCLUSION}

The transconjunctival approach for involutional entropion correction is as effective as the subciliary approach with lateral tarsal strip and retractor advancement procedure. The transconjunctival route achieved significantly shorter surgical time as compared to the subciliary approach.

\section{ETHICALAPPROVAL:}

The study was approved by the Ethical Review Committee of the Combined Military Hospital, Jhelum, Pakistan.

\section{PATIENTS' CONSENT:}

Written informed consents were obtained from all patients.

\section{CONFLICT OF INTEREST:}

The authors declared that they have NO affiliations with or involvement in any organisation or entity with any financial interest or non-financial interest in subject matter or material discussed in this manuscript.

\section{AUTHORS' CONTRIBUTION:}

FAK: Concept, study design and critical revision.

MFH: Data acquisition, analysis and interpretation.

SPKN: Finalapproval of the manuscript.

UTM: Accountable for all aspects of the manuscript.

\section{REFERENCES}

1. Chan D, Sokoya M, Ducic Y. Repair of the malpositioned lower Lid. Facial Plast Surg 2017; 33(6): 598 605. doi:10.1055/s-0037-1608711.

2. Han J, Lee SH, Shin HJ. Mini-incisional entropion repair for correcting involutional entropion, Full description and surgical outcome. Medicine 2019; 98(33):e16731. doi: 10.1097/MD.0000000000016731.

3. Entropion. In: Dutton JJ, editor Atlas of Oculoplastic and Orbital Surgery $1^{\text {st }}$ ed. China: Lippincott Williams \& Wilkins 2013;p. 124.

4. Lin P, Kitaguchi Y, Mupas-Uy J, Sabundayo MS, Takahashi Y, Kakizaki H. Involutional lower eyelid entropion: Causative factors and therapeutic management. Int Ophthalmol 2019; 39(8):1895-1907. doi: 10.1007/s10792-018-1004-1.

5. Cheung JJC, Wong CKH, Cheung LTY. Combined pentagonal resection and inferior retractor plication in involutional entropion. BMC Ophthalmol 2018; 18(1):329. doi: 10.1186/s12886-018-0986-9.

6. Ranno S, Sacchi M, Gilardi D, Lembo A, Nucci P. Retractor plication versus retractor plication and lateral tarsal strip for eyelid entropion correction. Eur J Ophthalmol 2014; 
24(2):141-6. doi: 10.5301/ejo.5000369.

7. Gigantelli JW. Entropion. In: Yanoff M, Duker JS, editor Ophthalmology $4^{\text {th }}$ ed. China: Elsevier Saunders 2014: p. 1280 .

8. Hooshangi H, Sezavar M, Bohluli B, Sarkarat F, Nasrollahi F, Seyedabbaszadeh S. Comparison of two transconjunctival and transcutaneous approaches in the incidence of sclera show during lower eyelid blepharoplasty. Biosci Biotech Res Comm 2017; 10(2): 102-7.

9. Kreis AJ, Shafi F, Madge SN. Transconjunctival entropion repair - the backdoor approach. Orbit 2013; 32(5):271-4. doi: 10.3109/01676830.2013.815230.

10. Erb MH, Uzcategui N, Dresner SC. Efficacy and complications of the transconjunctival entropion repair for lower eyelid involutional entropion. Ophthalmology 2006; 113(12):2351-6. doi: 10.1016/j.ophtha.2006.07.034.

11. Lee $H$, Takahashi $Y$, Ichinose A, Kakizaki H. Comparison of surgical outcomes between simple posterior layer advancement of lower eyelid retractors and combination with a lateral tarsal strip procedure for involutional entropion in a Japanese population. Br J Ophthalmol 2014; 98(11):1579-82. doi: 10.1136/bjophthalmol-2013-304830.

12. Ishida Y, Takahashi Y, Kakizaki H. Posterior layer advancement of lower eyelid retractors with transcanthal canthopexy for involutional lower eyelid entropion. Eye 2016; 30(11):1469-74. doi: 10.1038/eye.2016.150.

13. Boboridis KG, Bunce C. Interventions for involutional lower lid entropion. Cochrane Database Syst Rev 2011; (12): CD002221. doi: 10.1002/14651858.CD002221.pub2.

14. Nakos EA, Boboridis KG, Kakavouti-Doudou AA, Almaliotis DD, Sioulis CE, Karampatakis VE. Randomised controlled trial comparing everting sutures with a lateral tarsal strip for involutional lower eyelid entropion. Ophthalmol Ther 2019; 8(3): 397-406. doi: 10.1007/s40123-019-0189-3.

15. Mcveigh KA, Harrison R, Ford R. Entropion and ectropion repair: a snapshot of surgical practice in the United Kingdom. Orbit 2018; 37(2):105-9. doi: 10.1080/ 01676 830.2017.1383461.

16. Vahdani K, Ford R, Garrott H, Thaller VT. Lateral tarsal strip versus Bick's procedure in correction of eyelid malposition. Eye 2018; 32(6):1117-22. doi: 10.1038/s41433-018-
0048-9.

17. Marcet MM, Phelps PO, Lai JSM. Involutional entropion. Risk factors and surgical remedies. Current Opinion in Ophthalmology 2015; 26(5):416-21. doi: 10.1097/ICU. 0000000000000186.

18. Poon JS, Vahdani K, Thaller VT. Comparison of four combined procedures for correction of involutional lower eyelid entropion. J Craniofac Surg 2019; 30(4):1239-44. doi: 10.1097/SCS.0000000000005466.

19. Baek JS, Choi SC, Jang SY, Lee JH, Choi HS. Comparison of surgical outcome between quickert suture and quickert suture with modified lateral tarsal strip in involutional lower eyelid entropion. J Craniofac Surg 2016; 27(1):198-200. doi: 10.1097/SCS.0000000000002287.

20. Serin D, Buttanri IB, Karslioglu S, Sevim MS, Buttanri B, Akbaba M. The efficacy of the combined procedure in involutional entropion surgery: A comparative study. Korean J Ophthalmol 2013; 27(6):405-408. doi: 10.3341/ kjo.2013.27.6.405.

21. Boboridis KG, Mikropoulos DG, Ziakas NG. Entropion. Ophthalmology 2011; 118(1):225-6.

22. El-Samkary MA. Lower lid retractors tucking with gray-line splitting procedure in the management of lower eyelid involutional entropion with misdirected lashes. J Egypt Ophthalmol Soc 2018; 111:132-136. Doi: 10.4103/ejos. ejos_36_18.

23. lyengar SS, Dresner SC. Entropion. In: Black EH, Nesi FA, Calvano CJ, Gladstone GJ, Levine MR editors. Smith and Nesi's ophthalmic plastic and reconstructive surgery. 3rd ed. New York (NY): Springer 2012; p.311-315.

24. Ben Simon GJ, Molina M, Schwarcz RM, McCann JD, Goldberg RA. External (subciliary) vs. internal (transconjunctival) involutional entropion repair. Am J Ophthalmol 2005; 139(3):482-7. doi: 10.1016/j.ajo.2004. 10.003.

25. Goel R, Sanoria A, Kumar S, Arya D, Nagpal S, Rathie N. Comparison of polypropylene sling with combined transconjunctival retractor plication and lateral tarsal strip for correction of involutional lower eye lid ectropion. Open Ophthalmol J 2017; 2:285-97. doi: 10.2174/187436410 1711010285. 